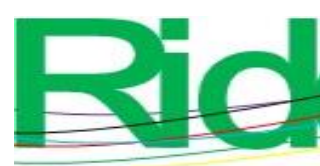

\title{
Determinantes de la inserción laboral en egresados universitarios en México
}

\author{
Determinants of Labor Insertion in University Graduates in México
}

\section{Determinantes da inserção profissional em graduados universitários no \\ México}

\author{
Omar Alejandro Pérez Cruz \\ Universidad de Colima, México \\ omar_perez@ucol.mx \\ https://orcid.org/0000-0003-3367-8259
}

Rogelio Pinto Pérez

Instituto de Estudios Universitarios, Plantel Puebla, México

rogepipe@ucol.mx

https://orcid.org/0000-0003-30579-6602

\section{Resumen}

La diversidad de cambios económicos, tecnológicos y sociales que se han experimentado en la última década ha influido en el mercado laboral, en las formas de entender la configuración y la dinámica del empleo, en los procesos de transición entre educación y trabajo, en las estrategias de formación, así como en los progresivos ajustes entre los planes curriculares de las instituciones de educación superior y la iniciación e inserción de los egresados al mercado laboral. Este artículo expone una revisión de algunos de los factores centrales en torno a la relación entre la educación superior y el mundo laboral, con la finalidad de contextualizar la empleabilidad como concepto, práctica e instrumento de la política económica y educativa en México. El objetivo fue estimar la significatividad estadística que un conjunto de variables predictivas (titulación, elementos académicos, elementos curriculares y habilidades personales) ejerce sobre la dependiente (inserción laboral). Para tal fin, se ha implementado 


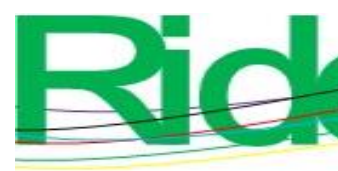

Revista Iberoamericana para la
Investigación y el Desarrollo Educativo
ISSN $2007-7467$

un estudio de encuesta mediante una estrategia mixta (online y telefónica), al igual que un análisis econométrico, con datos panel y regresión de mínimos cuadrados ordinarios (MCO) con errores estándar corregidos. Los resultados muestran que los titulados, y más los titulados por el Examen General para el Egreso de Licenciatura (EGEL), se asocian significativamente con la inserción laboral. Asimismo, las variables de emprendimiento, trabajo en equipo, ambiente de trabajo, prácticas de talleres y laboratorios y manejo de las tecnologías de la información y comunicación (TIC) se asocian significativamente con la inserción laboral. Al gestionar estas variables, es posible incrementar la probabilidad de los egresados de insertarse en el mercado laboral.

Palabras clave: datos panel, inserción laboral, seguimiento de egresados.

\section{Abstract}

The diversity of economic, technological and social changes that the world has been experienced in the last decade has influenced the labor market, the ways of understanding the configuration and dynamics of employment, the processes of transition between education and work, the training strategies, as well as the progressive adjustments between the curricula of universities and the initiation and insertion of graduates into the labor market. This article presents a review of some of the central factors around the relationship between higher education and the world of work, in order to contextualize employability as a concept, practice and instrument of economic and educational policy in Mexico. The objective was to estimate the statistical significance that a set of predictive variables (degree, academic elements, curricular elements and personal skills) exert on the dependent (labor insertion). To this end, a survey study has been implemented through a mixed strategy (online and telephone), as well as an econometric analysis, with panel data and ordinary least squares (OLS) regression with corrected standard errors. The results show that graduates, and more those entitled by exam, are significantly associated with labor insertion. Likewise, the variables of entrepreneurship, teamwork, work environment, workshop and laboratory practices, and ICT management are significantly associated with job placement. By managing these variables, it is possible to increase the probability of graduates to enter the labor market.

Keywords: panel data, labor insertion, follow-up of graduates. 


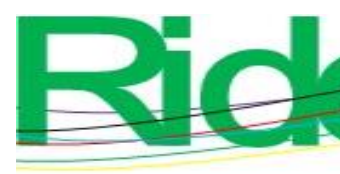
Revista Iberoamericana para la
Investigación y el Desarrollo Educativo
ISSN $2007-7467$

\section{Resumo}

A diversidade das mudanças económicas, tecnológicas e sociais vividas na última década tem influenciado o mercado de trabalho, nas formas de compreender a configuração e dinâmica do emprego, nos processos de transição entre educação e trabalho, nas estratégias de formação, bem como nos ajustes progressivos entre os planos curriculares das instituições de ensino superior e a iniciação e inserção dos licenciados no mercado de trabalho. Este artigo apresenta uma revisão de alguns dos fatores centrais em torno da relação entre o ensino superior e o mundo do trabalho, a fim de contextualizar a empregabilidade como conceito, prática e instrumento de política econômica e educacional no México. O objetivo foi estimar a significância estatística que um conjunto de variáveis preditivas (titulação, elementos acadêmicos, elementos curriculares e habilidades pessoais) exerce sobre o dependente (inserção laboral). Para tal, foi implementado um estudo survey, com estratégia mista (online e telefone), bem como uma análise econométrica, com dados em painel e regressão de mínimos quadrados ordinários (MQO) com erros padrão corrigidos. Os resultados mostram que os graduados, e mais os graduados pelo Exame Geral de Bacharelado (EGEL), estão significativamente associados à colocação profissional. Da mesma forma, as variáveis de empreendedorismo, trabalho em equipe, ambiente de trabalho, oficinas e práticas laboratoriais e gestão de tecnologias de informação e comunicação (TIC) estão significativamente associadas à colocação profissional. Com o gerenciamento dessas variáveis, é possível aumentar a probabilidade de os graduados ingressarem no mercado de trabalho.

Palavras-chave: dados de painel, colocação de emprego, monitoramento de graduação.

Fecha Recepción: Febrero 2020

Fecha Aceptación: Septiembre 2020 


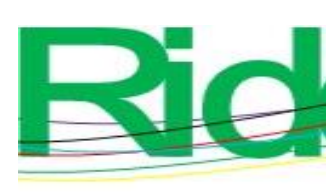

Revista Iberoamericana para la Investigación y el Desarrollo Educativo ISSN $2007-7467$

\section{Introducción}

La multiplicidad de avances tecnológicos y económicos que han experimentado las sociedades en las últimas décadas ha influido en la manera de concebir el mercado laboral, las formas de entender los procesos de formación y preparación para el ámbito laboral, las habilidades y destrezas profesionales que el sector empresarial demanda, así como en la paulatina estructuración de programas educativos para los diferentes perfiles académicos.

A nivel nacional, la progresiva aceleración de la dinámica laboral continuará en aumento en la medida en que se integren los mercados en Norteamérica y en la cuenca del Pacífico, y se incremente la oferta y demanda de talento humano especializado en el contexto comercial de los tratados comerciales que mantiene México.

Las instituciones de educación superior, en tanto organizaciones responsables de formar el talento humano calificado (y que, por lo tanto, integran las estadísticas de la población económicamente activa), se ven constantemente presionadas a actualizar sus programas de estudio para adecuarlos a contextos más regionales: los programas se moldean según las exigencias actuales del mercado laboral y los requerimientos futuros de cada profesión. En este sentido, la actualización de los programas de estudio demanda que se analice información objetiva sobre las trayectorias escolares de los estudiantes, desde su ingreso al nivel educativo hasta su egreso, titulación y seguimiento luego de uno, tres y cinco años de egresar. De forma tal que se obtengan datos objetivos sobre el proceso de inserción laboral, los cuales deberán ser tomados en cuenta al momento de redefinir el rumbo estratégico de las organizaciones educativas.

Teniendo en cuenta lo anterior, investigadores y académicos han comenzado a generar evidencia sobre los factores que influyen en la inserción laboral de los recién egresados de las instituciones de educación superior. Este artículo se inscribe en esa línea, ya que tiene el objetivo de estimar la significatividad estadística que un conjunto de variables predictivas (titulación, elementos académicos, elementos curriculares y habilidades personales) ejerce sobre la dependiente (inserción laboral).

La problemática aquí en cuestión se abordó en el siguiente orden. En primer lugar, se señalan los factores que han constituido, al menos parcialmente, la práctica de la inserción laboral bajo escenarios de desempleo funcional y estructural. En segundo lugar, se analizó la relación entre la inserción laboral y la educación. Enseguida, se expone la metodología de mínimos cuadrados ordinarios (MCO), la cual es aplicada a una muestra de egresados de una 


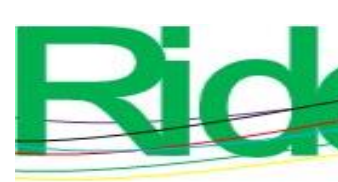

Revista Iberoamericana para la Investigación y el Desarrollo Educativo ISSN 2007 - 7467

problemática exclusiva de los recién egresados, conlleva profundas deudas entre la formación académica y el sistema económico, lo que implica déficit en la empleabilidad, y en especial, la ampliación de las limitantes para la inserción laboral de los egresados (Piana, 2019).

Estas barreras son resultado, además de la vorágine tecnológica que subyace en el desarrollo económico, de la tendencia empresarial de endurecer los criterios de selección para la ocupación de sus vacantes laborales (Ribes, 2017). Tal es el caso de algunos países de Latinoamérica. Así pues, la oferta de habilidades que los egresados han desarrollado en su formación se encuentra desfasada con respecto a las demandas del mercado laboral. Y estos desajustes entre el sistema formativo y las demandas laborales han conducido al desempleo de los egresados (De La Hoz, Quejada y Yánez, 2012).

\section{Inserción laboral y educación}

Los estudiantes que egresan e intentan incursionar en el mercado laboral por primera vez no reflexionan sobre las barreras de entrada y lo que conlleva insertarse en la población económicamente activa. De acuerdo con Chung (2019), en esta etapa de transición los jóvenes enfrentan una serie de dificultades que demanda la puesta en acción de un abanico de habilidades y competencias específicas necesarias para poder capitalizar de manera eficiente el aprendizaje adquirido a lo largo de cuatro o cinco años de formación. Si bien no hay fórmulas predeterminadas para garantizar la obtención de un empleo, sí existen indicadores más o menos significativos que guían los esfuerzos para la obtención de un espacio en el mercado laboral, o por lo menos, que aumentan la probabilidad de obtener dicho espacio. Al respecto, Bourdieu (1986) señala que son tres los tipos de capitales que contribuyen al éxito laboral: capital económico, social y académico (incorporado, objetivado e institucionalizado).

En este orden de ideas, Martínez y González (2019) señalan que los procesos de inserción laboral de los egresados de las instituciones de educación superior representan un campo de estudios altamente pertinentes, aunque pobremente analizada. Como parte de la literatura actual, se encuentran investigaciones de dicho tópico desde diversas perspectivas. Desde una de clases sociales, por ejemplo, Fachelli y Navarro (2015) aportan evidencia sobre el origen social y la inserción laboral. Rodríguez (2018) analizó los determinantes culturales y del capital humano del ingreso de las mujeres al mercado laboral. Mientras que Martínez y 


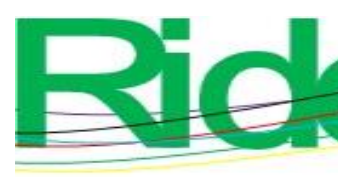

Revista Iberoamericana para la Investigación y el Desarrollo Educativo ISSN $2007-7467$

En cuanto a la variable dependiente, el modelo que se estimó aquí consideró la siguiente: Inserción laboral. Mientras que las variables independientes fueron: Titulación, Factores profesionales, Curriculares y Docentes, las cuales se muestran en la tabla 1.

Tabla 1. Descripción de las variables

\begin{tabular}{|l|l|l|}
\hline \multicolumn{1}{|c|}{ Dimensión } & \multicolumn{1}{|c|}{ Clave } & \multicolumn{1}{c|}{ Descripción } \\
\hline Año & AO & Del año al año (XXXX-XXXX). \\
\hline Inserción laboral & Emp 02 & Trabaja o no trabaja. \\
\hline Titulación & Tit01 y 02 & $\begin{array}{l}\text { Titulado: } a \text { ) Sí b) No } \\
\text { Titulado por Examen General para el } \\
\text { Egreso de Licenciatura (EGEL), } \\
\text { tesis, promedio. }\end{array}$ \\
\hline $\begin{array}{l}\text { Factores } \\
\text { profesionales }\end{array}$ & $\begin{array}{l}\text { Dp 101 al } \\
111\end{array}$ & $\begin{array}{l}\text { La puesta en práctica de los } \\
\text { conocimientos adquiridos. }\end{array}$ \\
\hline Factores curriculares & $\begin{array}{l}\text { Dp 201 al al } \\
220\end{array}$ & $\begin{array}{l}\text { Contenidos teóricos y prácticos en } \\
\text { escenarios académicos y reales. }\end{array}$ \\
\hline Factores docentes & $\begin{array}{l}\text { Dp 301 al } \\
314\end{array}$ & $\begin{array}{l}\text { Aspectos de los profesores que se } \\
\text { relacionan con la carrera. }\end{array}$ \\
\hline
\end{tabular}

Fuente: Elaboración propia

También es importante mencionar que la variable dependiente nominal contó con dos opciones de respuesta, esto es, era de carácter dicotómico. Como el interés de este estudio es estimar la significatividad estadística asociada a trabajar o no a partir de un conjunto de variables independientes, se optó por un análisis econométrico, con datos panel y MCO con errores estándar corregidos.

Esta metodología estadística para medir la correspondencia entre el nivel de educación y la participación laboral es el modelo tipo panel. De esta forma, se estructuraron los datos estadísticos en un panel, donde la unidad de observación se estructura en dos dimensiones, una de tipo transversal y otra por series de tiempo. Este tipo de diseño estadístico mejora la fiabilidad de las estimaciones al ampliar el número de observaciones que se analizan respecto a las generaciones de egresados del programa educativo estudiado, lo que permite un mayor control de la hipótesis. En este sentido, y retomando la información presentada en la tabla anterior, el modelo lineal de regresión múltiple se estableció de la siguiente manera:

Inserción laboral

$$
\begin{aligned}
& =\alpha+\beta_{1} \text { titulación }_{i t}+\beta_{2} \text { factores profesionales }_{i t} \\
& +\beta_{3} \text { factores curriculares }_{i t}+\beta_{4} \text { factores docentes }_{i t}
\end{aligned}
$$


Donde $i=1,2,3 \ldots 32$ y $t=2014,2005,2016,2017$.

Una vez establecidas las variables, se corrió un primer análisis con diversas variables independientes para verificar la significatividad con la dependiente. De las correlaciones obtenidas, se eliminaron aquellas variables independientes que no guardaban correlación con la dependiente. En la tabla 2, se muestra el modelo final.

Tabla 2. Descripción de las variables del modelo final

\begin{tabular}{|c|c|c|c|}
\hline Dimensión & Clave & Indicador & Descripción \\
\hline Año & $\mathrm{AO}$ & & Del año al año (XXXX-XXXX). \\
\hline $\begin{array}{l}\text { Inserción } \\
\text { laboral }\end{array}$ & Emp 02 & & Trabaja o no trabaja. \\
\hline Titulación & $\begin{array}{l}\text { Tit01 y } \\
02\end{array}$ & & $\begin{array}{l}\text { Titulado: } a) \text { Sí } \quad \text { b) No } \\
\text { Titulado por EGEL, tesis, promedio. }\end{array}$ \\
\hline \multirow[t]{3}{*}{$\begin{array}{l}\text { Factores } \\
\text { profesionale } \\
\mathrm{S}\end{array}$} & Dp 102 & Emprendimiento & $\begin{array}{l}\text { La capacidad para iniciar, mantener o } \\
\text { poner en práctica ideas propias para la } \\
\text { obtención de productos o servicios. }\end{array}$ \\
\hline & Dp 104 & $\begin{array}{l}\text { Trabajo } \\
\text { equipo }\end{array}$ & $\begin{array}{l}\text { La capacidad para } \\
\text { coordinadamente con } \\
\text { proyectos conjuntos. }\end{array}$ \\
\hline & Dp 108 & $\begin{array}{l}\text { Ambiente de } \\
\text { trabajo }\end{array}$ & $\begin{array}{l}\text { La capacidad para trabajar } \\
\text { armoniosamente con otras personas en } \\
\text { proyectos conjuntos. }\end{array}$ \\
\hline $\begin{array}{l}\text { Factores } \\
\text { curriculares }\end{array}$ & Dp 215 & $\begin{array}{l}\text { Manejo del } \\
\text { riesgo }\end{array}$ & $\begin{array}{l}\text { La disposición para asumir y administrar } \\
\text { riesgos controlados. }\end{array}$ \\
\hline \multirow[t]{3}{*}{$\begin{array}{l}\text { Factores } \\
\text { docentes }\end{array}$} & Dp 304 & $\begin{array}{lr}\text { Prácticas } & \text { de } \\
\text { talleres } & y \\
\text { laboratorios } & \end{array}$ & $\begin{array}{l}\text { Aspectos de los profesores que se } \\
\text { relacionan con la carrera. }\end{array}$ \\
\hline & & $\begin{array}{l}\text { Manejo de las } \\
\text { Tecnologías de } \\
\text { la información y } \\
\text { la comunicación } \\
\text { (TIC) }\end{array}$ & $\begin{array}{l}\text { Conjunto de habilidades para el manejo de } \\
\text { recursos digitales. }\end{array}$ \\
\hline & & Liderazgo & $\begin{array}{l}\text { Conjunto de habilidades para influir sobre } \\
\text { personas o grupos. }\end{array}$ \\
\hline
\end{tabular}

Fuente: Elaboración propia

La obtención de la correlación estadística entre las variables permitió eliminar en lo posible las heterogeneidades no observables del panel de datos, tratando de controlar el carácter individual de cada ente, y corregir, cuando así fue necesario, los problemas de heterocedasticidad y autocorrelación que se presentaron en el modelo final. De este modo, se llegó a los siguientes resultados. 


\section{Resultados}

Para corregir el problema de autocorrelación y heterocedasticidad que se presentó y para obtener una mayor confiabilidad en los resultados, se aplicó el estimador de errores estándar corregidos para panel (PCSE, por sus siglas en inglés), el cual se muestra en la siguiente tabla 3.

Tabla 3. Participación laboral en México

\begin{tabular}{|l|l|l|}
\hline Variable dependiente & & \\
\hline Titulación & & \\
\hline Variable independiente & Coeficiente & Std. Err. \\
\hline Titulado & 0.1015 & 0.0568 \\
\hline Titulado por EGEL & 0.1570 & 0.0523 \\
\hline
\end{tabular}

* Significativa $1 \%$. ** Significativa $5 \%$. ** Significativa $10 \%$.

Fuente: Elaboración propia

En la tabla anterior se puede observar que ambas variables, Titulación y Titulación por EGEL, resultaron significativas.

La tabla 4, muestra el siguiente análisis, el cual estimó la correlación de la misma variable dependiente con otras independientes.

Tabla 4. Participación laboral en México

\begin{tabular}{|l|l|r|r|}
\hline & Variable dependiente & & \\
\hline & Inserción laboral & & \\
\hline Factores & Variable independiente & \multicolumn{1}{|c|}{ Coeficiente } & \multicolumn{1}{|c|}{ Std. Err. } \\
\hline Profesionales & Emprendimiento & $0.2361^{* *}$ & 0.0993 \\
\hline Profesionales & Trabajo en equipo & $0.3776^{*}$ & 0.1057 \\
\hline Profesionales & Ambiente de trabajo & $0.2950^{*}$ & 0.0976 \\
\hline Curriculares & Disposición para el manejo del riesgo & 0.1177 & 0.0902 \\
\hline Docentes & Prácticas de talleres y laboratorios & $0.2215^{*}$ & 0.0812 \\
\hline Docentes & Manejo de TIC & $0.2214^{* *}$ & 0.1043 \\
\hline Docentes & Liderazgo & 0.1819 & 0.0681 \\
\hline
\end{tabular}

* Significativa $1 \%$. ** Significativa $5 \%$. *** Significativa $10 \%$.

Fuente: Elaboración propia 


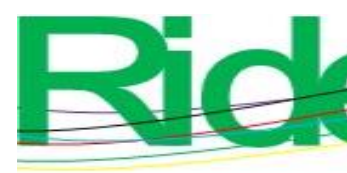

Revista Iberoamericana para la Investigación y el Desarrollo Educativo ISSN $2007-7467$

Respecto a la tabla 4, se puede observar que los factores que resultaron significativos fueron los englobados en Profesionales y Docentes.

De manera individual, los indicadores que integran los factores que obtuvieron valores significativos fueron: Emprendimiento, Trabajo en equipo, Ambiente de trabajo, Prácticas de talleres y laboratorios y Manejo de TIC.

Los indicadores de Emprendimiento, Ambiente de trabajo y Prácticas de talleres y laboratorios resultaron con tendencia positiva. Esto explica que cuando los egresados emprenden ideas propias para productos o servicios se integran más rápidamente al mercado laboral, lo que concuerda con los resultados de García (2015). Asimismo, Ambiente de trabajo y Prácticas realizadas en talleres y laboratorios incrementan la posibilidad de insertarse en el mercado de trabajo, resultados que están en consonancia, por su parte, con los de Gallegos (2005).

Por otro lado, las variables Trabajo en equipo y Manejo de TIC guardan también una relación significativa y con tendencia positiva. Esto explica que a mayor de estas competencias mayor posibilidad de inserción laboral. Los resultados de González, Vila y Guisado (2016) comparten esta visión.

Finalmente, los resultados también permiten mostrar que es necesario que se ajuste el plan de estudio de la licenciatura en Medicina, para que los contenidos curriculares impacten en la inserción laboral de sus egresados. Para esto se requiere el apoyo del claustro académico, el cual debe velar por el fortalecimiento de las habilidades profesionales de sus estudiantes.

\section{Discusión}

La finalidad de esta investigación fue estimar la significatividad estadística que un conjunto de variables predictivas (titulación, elementos académicos, elementos curriculares y habilidades personales) ejerce sobre la dependiente (inserción laboral). Para lo cual se diseñaron cuatro hipótesis.

La primera hipótesis relaciona la inserción laboral con los estudiantes que se titularon, la cual se aceptó. Esto explica que la titulación influye en la inserción laboral; aún más significativo resulta haberse titulado por el EGEL, ya que dicho examen permite conocer el nivel de conocimientos y habilidades de los egresados de licenciatura, con base en pruebas estandarizadas por el Centro Nacional de Evaluación para la Educación Superior (Ceneval). 


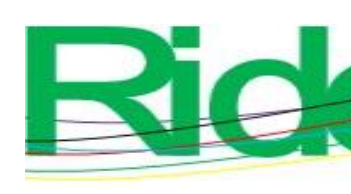

Revista Iberoamericana para la
Investigación y el Desarrollo Educativo
ISSN $2007-7467$

Barrera, Canepa y Santiago (2016) muestran resultados similares. En el caso de la muestra analizada en esta investigación, resulta especialmente significativo que los titulados tengan trabajo, por las regulaciones sanitarias y profesionales que demanda el Estado, además de la cultura profesional de los médicos. Este resultado coincide con el de Cabello (2016) y el de Vargas y Rivera (2006), quienes trabajaron con estudiantes de odontología.

Las hipótesis dos (factores profesionales) y cuatro (factores docentes) también fueron aceptadas. Estos resultados están en consonancia con Martínez y González (2019), quienes explican la relevancia de los aspectos profesionales (competencias personales) y docentes (vinculadores). Igualmente, Rivas, Cardoso y Cortés (2019) explican cómo estos influyen en el proceso de inserción laboral actualmente.

Estos resultados evidencian, además, que las universidades deben gestionar la empleabilidad por medio de estrategias orientadas al impulso de este tipo de factores. Así, se promoverá una participación social real que fortalezca la relación universidad-mercado laboral y, por extensión, abrirá mayores oportunidades de trabajo y perfiles objetivos de trabajo a largo plazo. Sin duda, los resultados aquí obtenidos son de interés para académicos, investigadores y personas que elaboran políticas públicas.

\section{Conclusiones}

Analizar los efectos de la correspondencia entre la inserción laboral y la educación dando seguimiento a egresados implica partir de la diversidad de las competencias profesionales.

El objetivo de esta investigación fue estimar la significatividad estadística que un conjunto de variables predictivas (titulación, elementos académicos, elementos curriculares y habilidades personales) ejerce sobre la inserción laboral. La inserción laboral es un tema que genera mayor preocupación desde el papel de las universidades en la determinación del desarrollo social en México.

De los resultados arrojados en esta investigación, se muestra claramente que las hipótesis planteadas por las estimaciones desarrolladas proporcionan evidencia empírica suficiente sobre el papel relevante de las universidades e instituciones de educación superior en la empleabilidad nacional, situación que es retomada en diversas investigaciones ya referidas. 


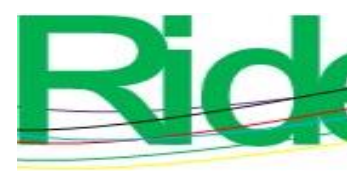

Revista Iberoamericana para la
Investigación y el Desarrollo Educativo
ISSN $2007-7467$

Para finalizar, a partir de los resultados encontrados en este análisis empírico de la función de las universidades, se verificó que los factores de titulación, profesionales, académicos y curriculares impactan de manera positiva en la inserción laboral de los médicos del país, en este caso en específico. Sin embargo, y aun cuando estos factores resultan significativos, los esfuerzos sobre inserción laboral y seguimiento de egresados todavía son incipientes en México, por lo que, a partir de este trabajo, se plantean diversas líneas futuras de investigación que sirvan de guía para modelar su impacto en la educación del país.

\section{Referencias}

Antunes, R. y Pochmann, M. (2008). La desconstrucción del trabajo y la explosión del desempleo estructural y de la pobreza en Brasil. En Cimadamore, A. D. y Cattani, A. D. (coords.), Producción de pobreza y desigualdad en América Latina (pp. 191-204). Bogotá, Colombia: Siglo del Hombre Editores. Recuperado de http://bibliotecavirtual.clacso.org.ar/ar/libros/clacso/crop/cattani/cattani.pdf.

Barrera, R. A., Canepa, A. A. y Santiago, J. C. (2016). Desempeño de egresados de tecnologías de información en el examen general de egreso. RIDE. Revista Iberoamericana para la Investigación y el Desarrollo Educativo, 7(13), 497-513. Recuperado de https://www.ride.org.mx/index.php/RIDE/article/view/261.

Bourdieu, P. (1986). The forms of capital. In Richardson, J., Handbook of Theory and Research for the Sociology of Education (pp. 241-258). New York, United States: Greenwood Press.

Cabello, N. (10 de mayo de 2016). Carreras de salud suben 50\% su matrícula, pero la mayoría tiene baja tasa de titulación. El Mercurio.

Cárdenas, J., Guataquí, J. y Montaña, J. (2015). Metodología para el análisis de demanda laboral mediante datos de internet: el caso colombiano. Revista de Economía del Rosario, 18(1), 93-126.

Chung, S. (2019). El efecto de la polarización del mercado de trabajo sobre la inserción laboral de los estudiantes universitarios en la República de Corea. Repercusiones en el desempleo juvenil. Revista Internacional del Trabajo, 138(3), 545-571. Recuperado de https://doi.org/10.1111/ilrs.12133. 


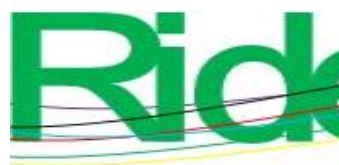

Revista Iberoamericana para la
Investigación y el Desarrollo Educativo
ISSN $2007-7467$

De La Hoz, F., Quejada, R. y Yánez, M. (2012). El desempleo juvenil: problema de efectos perpetuos. Revista Latinoamericana de Ciencias Sociales, Niñez y Juventud, 10(1), 427-439.

Recuperado

de http://www.umanizales.edu.co/publicaciones/campos/cinde/index.html.

Fachelli, S. and Navarro, J. (2015). Relationship between social origin and labor insertion of university graduates. Electronic Journal of Educational Research, Assessment and Evaluation, 21(2), 1-25. Retrieved from https://doi.org/10.7203/relieve.21.2.7812.

Gallegos, I. (2005). Seguimiento de egresados en relación al mercado de trabajo y práctica profesional de la carrera de cirujano dentista F.E.S. Zaragoza, periodo 1977 a 2002. (tesis). Universidad Nacional Autónoma de México, Ciudad de México.

García, D. (2015). Emprendimiento Empresarial Juvenil: Una evaluación con jóvenes estudiantes de universidad. Revista Latinoamericana de Ciencias Sociales, Niñez y Juventud, 13(2), 1221-1236.

González, M., Vila, M. y Guisado, T. (2016). Innovación, capacidad productiva, formación en el puesto de trabajo y productividad. Cuadernos de Gestión, 16(2), 77-92. Recuperado de https://doi.org/10.5295/cdg.140513mg.

Martínez, P. y González, C. (2019). Competencias personales y participativas vinculantes a la inserción laboral de los universitarios. Revista Electrónica de Investigación y Evaluación Educativa, 25(1), 1-35. Recuperado de https://doi.org/10.7203/relieve.25.1.13164.

Martínez, F. y Carmona, G. (2010). Test de factores sociopersonales para la inserción laboral de los jóvenes: un instrumento para la evaluación y la formación. ESE: Estudios sobre Educación, 18, 115-138.

Piana, R. (2019). Procesos de selección de personal en la administración pública de la Provincia de Buenos Aires. Una elipsis al régimen de concursos para el acceso a las funciones jerarquizadas. Documentos y Aportes en Administración Pública y Gestión Estatal, $\quad$ 18(31), 81-110. Recuperado de https://doi.org/10.14409/daapge.v18i31.8449.

Real Academia Española [RAE]. (2019). Empleabilidad. Recuperado de https://dle.rae.es/empleabilidad. 

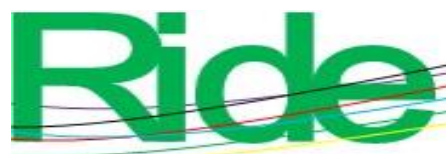

Revista Iberoamericana para la Investigación y el Desarrollo Educativo ISSN $2007-7467$

Ribes, M. (2017). Cuestiones críticas en los criterios de selección de trabajadores afectados por los despidos colectivos en el sector público. Lan Harremanak - Revista de Relaciones Laborales, 1(36), 191-215. Recuperado de https://doi.org/10.1387/lanharremanak.17932.

Rivas, R. M., Cardoso, E. O. y Cortés, J. A. (2019). Propuesta de las competencias profesionales en turismo desde el enfoque del empleador. RIDE Revista Iberoamericana para la Investigación y el Desarrollo Educativo, 9(18), 195-514. Recuperado de https://doi.org/10.23913/ride.v9i18.418.

Rodríguez, C. (2018). Capital humano y factores culturales: determinantes de la inserción laboral femenina en Chile. Perfiles Latinoamericanos, 26(52), 1-22. Recuperado de https://doi.org/10.18504/pl2652-008-2018.

Vargas, M. y Rivera, N. (2006). El examen de titulación en odontología como indicador de calidad profesional en la Universidad Michoacana de San Nicolás de Hidalgo, México. Revista Cubana de Educación Médica Superior, 20(3), 57-66.

Wikipedia. (23 de agosto de 2020). Desempleo. Recuperado de https://es.wikipedia.org/wiki/Desempleo\#Desempleo_c\%C3\%ADclico. 


\begin{tabular}{|c|c|}
\hline$\sum$ & $\begin{array}{l}\text { Revista Iberoamericana para la } \\
\text { Investigación y el Desarrollo Educativo } \\
\text { ISSN } 2007-7467\end{array}$ \\
\hline Rol de Contribución & Autor (es) \\
\hline Conceptualización & Omar Alejandro Pérez Cruz \\
\hline Metodología & Omar Alejandro Pérez Cruz \\
\hline Software & Omar Alejandro Pérez Cruz \\
\hline Validación & Rogelio Pinto Pérez \\
\hline Análisis Formal & Omar Alejandro Pérez Cruz \\
\hline Investigación & Rogelio Pinto Pérez \\
\hline Recursos & Rogelio Pinto Pérez \\
\hline Curación de datos & Rogelio Pinto Pérez \\
\hline $\begin{array}{l}\text { Escritura - Preparación del } \\
\text { borrador original }\end{array}$ & Omar Alejandro Pérez Cruz \\
\hline $\begin{array}{l}\text { Escritura - Revisión y } \\
\text { edición }\end{array}$ & Omar Alejandro Pérez Cruz \\
\hline Visualización & Omar Alejandro Pérez Cruz \\
\hline Supervisión & Omar Alejandro Pérez Cruz \\
\hline $\begin{array}{l}\text { Administración de } \\
\text { Proyectos }\end{array}$ & Rogelio Pinto Pérez \\
\hline Adquisición de fondos & Rogelio Pinto Pérez \\
\hline
\end{tabular}

